\title{
Large Intestinal Submucosa
}

National Cancer Institute

\section{Source}

National Cancer Institute. Large Intestinal Submucosa. NCI Thesaurus. Code C32930.

The layer of areolar tissue situated beneath the mucous membrane in the distal portion

of the digestive tract from the small intestine to the anus. 